\title{
Implantable collamer lens with a central hole for residual refractive error correction after corneal refractive surgery
}

\author{
JING ZHANG ${ }^{*}$, FANGLIN HE* ${ }^{*}$ YAN LIU and XIANQUN FAN \\ Department of Ophthalmology, Shanghai Key Laboratory of Orbital Diseases and Ocular Oncology, \\ Shanghai Ninth People's Hospital, Shanghai Jiao Tong University School of Medicine, Shanghai 200011, P.R. China
}

Received December 9, 2019; Accepted September 11, 2020

DOI: $10.3892 /$ etm.2020.9289

\begin{abstract}
The present study aimed to assess the visual and refractive outcomes of an implantable collamer lens with a central hole (ICL V4c) for residual refractive error correction after corneal refractive surgery in individuals with myopia. A total of 16 eyes of eight consecutive patients with myopia undergoing ICL V4c implantation after corneal refractive surgery were investigated. The uncorrected visual acuity (VA) and best-corrected VA were examined prior to surgery and at 1,3 and 6 months after surgery. The post-operative values of the modulation transfer function (MTF) cutoff frequency, Strehl ratio (SR), objective scattering index (OSI) and Optical Quality Analysis System (OQAS) values (OVs) were quantitatively assessed using an OQAS. At 6 months after surgery, the mean uncorrected LogMAR VA was $0.06 \pm 0.10$ and the values had improved in $100 \%$ of the eyes. The mean MTF cutoff frequency, SR, OSI, OV $100 \%$, OV $20 \%$ and OV 9\%, were $31.294 \pm 4.321$ cycles/degree, $0.187 \pm 0.039,1.399 \pm 0.274$, $1.066 \pm 0.261,0.748 \pm 0.287$ and $0.509 \pm 0.229$, respectively. In conclusion, ICL V4c implantation for the correction of residual refractive error after corneal refractive surgery appeared feasible and safe and also had an excellent optical performance. However, long-term changes in visual quality require further investigation.
\end{abstract}

\section{Introduction}

The Visian Implantable Collamer Lens (ICL ${ }^{\mathrm{TM}}$; STAAR Surgical) has been used for the correction of high myopia for

Correspondence to: Dr Xianqun Fan, Department of Ophthalmology, Shanghai Key Laboratory of Orbital Diseases and Ocular Oncology, Shanghai Ninth People's Hospital, Shanghai Jiao Tong University School of Medicine, 639 Zhi Zao Ju Road, Shanghai 200011, P.R. China

E-mail: fanxq@sjtu.edu.cn

*Contributed equally

Key words: implantable collamer lens with a central hole, visual quality, residual refractive error
$>10$ years (1-4). Particularly in recent years, with improvements in surgical techniques and the update of ICL design, it has been widely used. This surgical procedure is largely reversible, allowing the lens to be exchanged when refractive changes and unexpected complications occur after surgery. The currently used approaches for the correction of residual refractive error after corneal refractive surgeries mainly include corneal enhancement surgeries and relaxing corneal incisions (5-8). Considering the thinner corneal thickness after corneal refractive surgery, it is unsafe to perform corneal laser surgery again without sufficient residual stromal bed thickness. The safety of repeating corneal laser surgery therefore depends on the residual stromal bed thickness and the amount of residual correction required. Particularly in patients with large residual refractive error, the application of ICL has unique advantages.

Previous studies have demonstrated that ICL V4c implantation for correcting moderate to high myopia has good safety, efficacy, predictability and stability after long-term follow-up (9-11). Recently, ICL implantation has also been confirmed to perform well for low and moderate myopia $(12,13)$. These results provide theoretical support and guidance for the use of ICL to correct residual refractive errors after corneal refractive surgery.

In the present study, to evaluate the visual quality after ICL implantation, an Optical Quality Analysis System (OQAS; Terrassa, Spain), a device that employs the double-pass technique to assess the quality of retinal imaging, was used. Previously, it has been generally used to evaluate the visual quality after ICL implantation, which allows the detection of possible asymmetries in retinal images and objective measurement of ocular scatter (14-16).

In the present study, the safety and efficacy of ICL implantation for residual refractive errors after corneal laser surgery were evaluated by measuring the visual acuity and visual quality.

\section{Materials and methods}

Subjects. The present study included a total of 16 eyes from eight patients (three males and five females) who underwent implantation of a phakic posterior chamber ICL V4c for residual refractive error after corneal refractive surgeries between August 2015 and February 2018 at the Shanghai 
Ninth Hospital Affiliated with Jiao Tong University (Shanghai, China). Among these, four eyes of two patients were treated with photorefractive keratectomy (PRK) 20 years ago (June, 1998-October, 1999) and 12 eyes of six patients were treated with laser in situ keratomileusis (LASIK) 10 years ago (August, 2006-September, 2009). There was no intraoperative or postoperative complication during the first operation and the diopters of all patients were stable during the last 2 years.

All patients underwent a full ophthalmic evaluation pre-operatively and met the surgical requirements. The uncorrected visual acuity (UCVA), best-corrected visual acuity (BCVA), manifest refractive error, intraocular pressure (IOP; non-contact tonometer; Topcon), anterior chamber depth (ACD; Pentacam; Oculus), endothelial cell density (ECD; Topcon-SP), corneal topography (Pentacam), slit-lamp microscopy, funduscopic examination and visual quality (OQAS; Terrassa) measurements were performed prior to surgery.

Surgical scheme design. In view of the inherent measurement of refractive errors in eyes after corneal refractive surgery, the pre-operative subjective refraction was assessed using the same illumination conditions by two experienced residents who were trained to measure refractive errors using a comprehensive refractometer. The patient underwent small pupil refraction and cycloplegia refraction. In addition, considering the relatively advanced age of the patients, the diopters (D) were easily overcorrected after corneal refractive surgery; therefore, emmetropia was usually selected as the target refraction in the dominant eye and the target $\mathrm{D}$ was adjusted to retain $-0.50 \mathrm{D}$ in the non-dominant eye when the patients were $>45$ years of age.

Inclusion criteria. An anterior chamber depth of $\geq 2.80 \mathrm{~mm}$ and an endothelial cell density $>2,000$ cells $/ \mathrm{mm}^{2}$ were included in the inclusion criteria in the present study. It required that patients have a reasonable expectation of surgical outcomes and patients with keratoconus, cataract or glaucoma and systemic disease were excluded. The baseline data of all patients are listed in Table I.

Surgical procedure. All surgeries were performed by the same experienced surgeon (JZ). In the present study, the ICL model was ICL V4c with a $0.36-\mathrm{mm}$ central artificial hole (ICL V4c $\mathrm{c}^{\mathrm{TM}}$; STAAR Surgical). For ICL V4c implantation, without pre-operative peripheral iridotomy or intraoperative iridectomy, the procedure was different from the V4 surgery process. On the day of surgery, the pupils of patients were first enlarged. After topical anesthesia, a model V4c ICL was inserted into the anterior chamber through a 3-mm temporal clear corneal incision without sodium hyaluronate injection. When ICL V4c was injected into the anterior chamber, viscoelastic agent was injected into the surface of the ICL in the anterior chamber, and the ICL was then adjusted to enter the posterior chamber. Afterwards, the viscoelastic surgical agent was easily washed out of the anterior chamber using automatic irrigation/aspiration.

Postoperative follow-up. All surgeries were uneventful and no intraoperative complications occurred. After surgery, $0.1 \%$ tobramycin dexamethasone eye drops (Tobradex; Alcon) were prescribed three times daily for 1 week, after which the dose was tapered off over 2 weeks. Steroidal medication was
Table I. Characteristics of participants who underwent refractive error adjustment with the collamer lens implantation $(n=16$ eyes $)$.

\begin{tabular}{lc}
\hline A, General & \\
\hline Variable & Value \\
\hline Age (years) & \\
Mean \pm SD & $39.16 \pm 7.52$ \\
Range & $32-47$ \\
Sex (male/female) & $3 / 5$ \\
Safety indices & \\
Efficacy indices $^{\mathrm{b}}$ & $1.26 \pm 0.21$ \\
& $1.19 \pm 0.24$
\end{tabular}

$\mathrm{B}$, Pre- and post-operative parameters

\begin{tabular}{lcc}
\hline Variable & Pre-operative & $\begin{array}{c}\text { Post-operative } \\
(6 \text { months })\end{array}$ \\
\hline $\begin{array}{l}\text { Manifest spherical } \\
\text { equivalent (D) }\end{array}$ & $-4.26 \pm 1.55$ & $-0.53 \pm 0.12$ \\
Manifest cylinder (D) & $-0.75 \pm 0.23$ & $-0.37 \pm 0.09$ \\
LogMAR UCVA & $0.68 \pm 0.19$ & $0.06 \pm 0.10$ \\
LogMAR BCVA & $0.09 \pm 0.08$ & $-0.02 \pm 0.07$ \\
\hline
\end{tabular}

Values are expressed as the mean $\pm \mathrm{SD}$ or $\mathrm{n}$ unless otherwise speci-

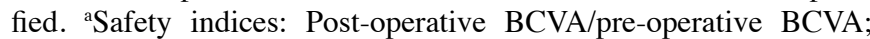
befficacy indices: Post-operative UCVA/pre-operative BCVA. D, Diopters; SD, standard deviation; Yrs, years; UCVA, uncorrected visual acuity; BCVA, best-corrected visual acuity.

provided at any time if the IOP monitoring suggested that it was required. Antibiotic medications (ofloxacin; Santen) were given four times daily for 1 week and artificial tears were administered four times daily for 2 months.

Prior to and 6 months after surgery, the UCVA, BCVA, Spherical equivalent (SE), IOP, ECD, and ACD were checked (Table II). The optical quality assessment was performed by OQAS, pre-operatively and 1,3 and 6 months post-operatively.

OQAS measurement. The objective scattering index (OSI), the values of modulation transfer function (MTF) cutoff frequency, the Strehl ratio (SR) and the OQAS values (OVs) were used to evaluate the visual performance. The meanings and calculations of the parameters were reported in several previous studies (14-16). The determination of the OSI is an objective evaluation of intraocular scattered light. The index is calculated by evaluating the amount of light outside the double-pass retinal intensity point spread function (PSF) image in relation to the amount of light in the center (14-16). The MTF cutoff value is the frequency at which the MTF reaches a value of 0.01 . It refers to the frequency, up to which the eye is able to focus an object on the retina with a significant $1 \%$ contrast. The three OVs are normalized values of three spatial frequencies, which correspond to MTF values that describe the optical quality of the eye for three contrast conditions, commonly used in ophthalmic practice: $100 \%(\mathrm{OV}$ 
Table II. Comparison pre- and postoperative parameters in both eyes undergoing implantable collamer lens (ICL) implantation after corneal refractive surgery.

\begin{tabular}{|c|c|c|c|c|c|c|}
\hline \multirow[b]{2}{*}{ Parameters } & \multicolumn{3}{|c|}{ OD (8 eyes) } & \multicolumn{3}{|c|}{ OS (8 eyes) } \\
\hline & Preoperative & Post 6 months & $\mathrm{P}$-value & Preoperative & Post 6 months & P-value \\
\hline Mean $\mathrm{SE}(\mathrm{D}) \pm \mathrm{SD}$ & $-4.40 \pm 1.78$ & $-0.19 \pm 0.23$ & $<0.01$ & $-4.15 \pm 1.61$ & $-0.13 \pm 0.18$ & $<0.01$ \\
\hline Log MAR UCVA & $0.53 \pm 0.19$ & $0.05 \pm 0.09$ & $<0.01$ & $0.44 \pm 0.19$ & $-0.04 \pm 0.05$ & $<0.01$ \\
\hline Log MAR BCVA & $0.08 \pm 0.14$ & $0.01 \pm 0.09$ & 0.02 & $0.00 \pm 0.01$ & $-0.05 \pm 0.05$ & 0.03 \\
\hline $\mathrm{IOP}(\mathrm{mmHg})$ & $11.95 \pm 2.03$ & $12.18 \pm 1.99$ & 0.18 & $11.54 \pm 1.58$ & $11.90 \pm 1.64$ & 0.12 \\
\hline $\operatorname{ECD}\left(\operatorname{cell} / \mathrm{mm}^{2}\right)$ & $2,674.25 \pm 110.56$ & $2,659.13 \pm 98.75$ & 0.05 & $2,683.00 \pm 99.02$ & $2,671.00 \pm 101.48$ & 0.06 \\
\hline $\mathrm{ACD}(\mathrm{mm})$ & $3.29 \pm 0.22$ & $3.31 \pm 0.21$ & 0.13 & $3.28 \pm 0.24$ & $3.31 \pm 0.23$ & 0.11 \\
\hline
\end{tabular}

OD, oculus dexter; OS, oculus sinister; D, Diopters; SD, standard deviation; SE, spherical equivalent; UCVA, uncorrected visual acuity; BCVA, best-corrected visual acuity; IOP, Intraocular pressure; ECD, endothelial cell density; ACD, Anterior chamber depth.

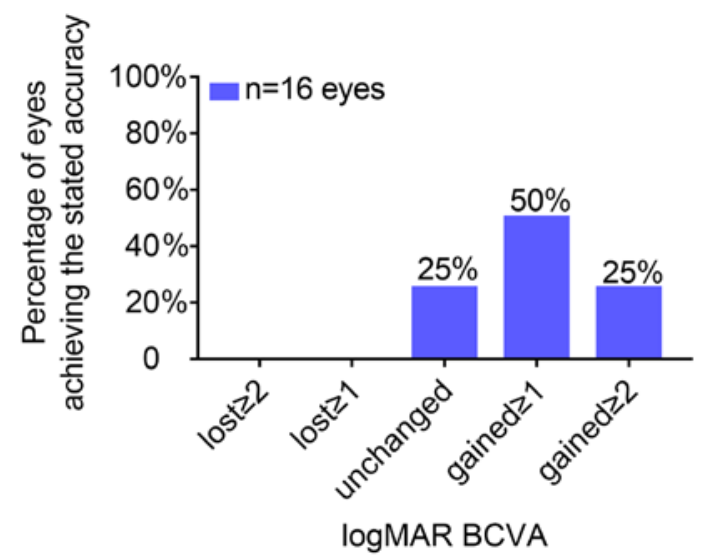

Figure 1. Changes in BCVA 6 months after implantable collamer lens implantation ( $\mathrm{n}=16$ eyes). BCVA, best-corrected visual acuity.

$100 \%$ ), 20\% (OV 20\%) and 9\% (OV 9\%). The SR is the ratio of the central maximum of the illuminance of the PSF in the aberrated eye to the central maximum that would be expected in a corresponding aberration-free system (14-16).

Statistical analysis. All statistical analyses were performed using SPSS version 20.0 (IBM Corp.) and the results are expressed as the mean \pm standard deviation. Repeated-measures analysis of variance was used to analyze the differences in UCVA, BCVA, IOP and ACD as well as ECD between the eyes prior to and after the operation. Pre-operative and post-operative visual quality was determined at different time-points by using repeated-measures analysis of variance, followed by Tukey's post hoc test. $\mathrm{P}<0.05$ was considered to indicate statistical significance. Pearson's correlation coefficient analysis was used to calculate the degree and significance of correlation between the achieved and predicted MRSE.

\section{Results}

Clinical features of patients. All surgeries were uneventful and no post-operative complications were observed during the 6-month follow-up. The pre-operative LogMAR UCVA and
BCVA was $0.68 \pm 0.19$ and $0.09 \pm 0.08$, respectively (Table I). After the ICL implantation, the LogMAR UCVA and BCVA improved significantly, to $0.06 \pm 0.10$ and $-0.02 \pm 0.07$, respectively $(\mathrm{P}<0.001)$. The mean values of manifest refractive spherical equivalent (Mean SE) changed from $-4.40 \pm 1.78 \mathrm{D}$ pre-operatively to $-0.19 \pm 0.23 \mathrm{D}$ in the right eye at post-operative 6 months and changed from $-4.15 \pm 1.61 \mathrm{D}$ pre-operatively to $-0.13 \pm 0.18 \mathrm{D}$ in the left eye at 6 months post-operatively (Table II). The parameters of binocular vision 6 months after surgery were compared and the differences in UCVA, BCVA and SE prior to and after surgery were statistically significant, but there was no significant difference in IOP, ECD and ACD prior to and after surgery (Table II).

Visual outcomes. At 6 months after surgery, none of the examined eyes exhibited a loss in BCVA of one line or more. Regarding BCVA, four eyes did not change after surgery, eight eyes gained one line and four eyes gained two lines (Fig. 1). The safety indices (mean post-operative BCVA/mean pre-operative BCVA) and the efficacy indices (mean postoperative UCVA/mean pre-operative BCVA) at 6 months were $1.26 \pm 0.21$ and $1.19 \pm 0.24$, respectively (Table I).

$O Q A S$ results. There was no statistically significant difference in the OSI, SR and OVs at contrasts of $9 \%$ at 1,3 and 6 months after ICL implantation, respectively. However, there were significant differences in the MTF cutoff frequency and OVs at contrasts of 100 and $20 \%$, pre-operatively and 1,3 and 6 months post-operatively (repeated-measures analysis of covariance; $\mathrm{P}<0.05)$. These results are summarized in Table III and Fig. 2.

Changes in MRSE. The deviation of the achieved MRSE from the predicted MRSE was determined. At 6 months, all eyes were within $0.50 \mathrm{D}$ of the target refractive change (Pearson's correlation, $\mathrm{R}=0.944$; $\mathrm{P}<0.0001$; Fig. 3).

\section{Discussion}

Posterior chamber phakic intraocular lens (pIOL) implantation is a safe and effective refractive surgery that has been widely accepted. Previous studies have demonstrated that ICL 
Table III. Optical quality parameters in eyes subjected to implantable collamer lens implantation after corneal refractive surgery.

\begin{tabular}{|c|c|c|c|c|c|c|c|}
\hline \multirow{3}{*}{$\begin{array}{l}\text { OQAS } \\
\text { parameter }\end{array}$} & \multirow[b]{3}{*}{ Pre-operative } & \multicolumn{3}{|c|}{ Post-operative } & \multicolumn{2}{|c|}{ P-value } & \multirow{3}{*}{$\begin{array}{l}\text { Pre-operative vs } \\
\text { post } 6 \text { months }\end{array}$} \\
\hline & & & & & Pre-operative & Pre-operative vs. & \\
\hline & & 1 month & 3 months & 6 months & vs. 1 month & post 3 months & \\
\hline MTF cutoff & 28.739 & 30.274 & 30.738 & 31.294 & $<0.001$ & $<0.001$ & $<0.001$ \\
\hline OSI & 1.550 & 1.516 & 1.469 & 1.399 & 0.165 & 0.153 & 0.142 \\
\hline SR & 0.183 & 0.171 & 0.175 & 0.187 & 0.089 & 0.104 & 0.130 \\
\hline OV100\% & 0.890 & 1.021 & 1.048 & 1.066 & $<0.001$ & $<0.001$ & $<0.001$ \\
\hline OV20\% & 0.654 & 0.701 & 0.723 & 0.748 & 0.008 & 0.006 & 0.005 \\
\hline OV9\% & 0.483 & 0.509 & 0.507 & 0.509 & 0.122 & 0.134 & 0.121 \\
\hline
\end{tabular}

MTF, modulation transfer function; SR, Strehl ratio; OSI, objective scattering index; OQAS, Optical Quality Analysis System; OV, OQAS value.

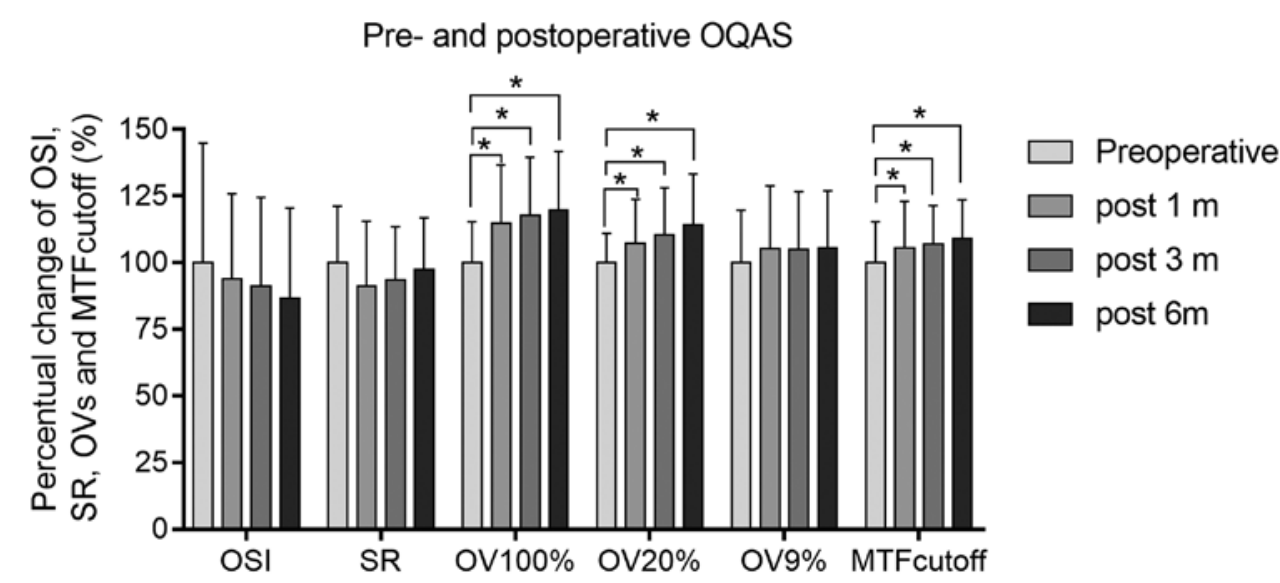

Figure 2. Pre- and postoperative optical quality parameters. The measured values were reflected as the percentual changes that were corrected to the preoperative group values by data normalization. ${ }^{*} \mathrm{P}<0.05$. MTF, modulation transfer function; SR, Strehl ratio; OSI, objective scattering index; OQAS, Optical Quality Analysis System; OV, OQAS value; mo, month(s).

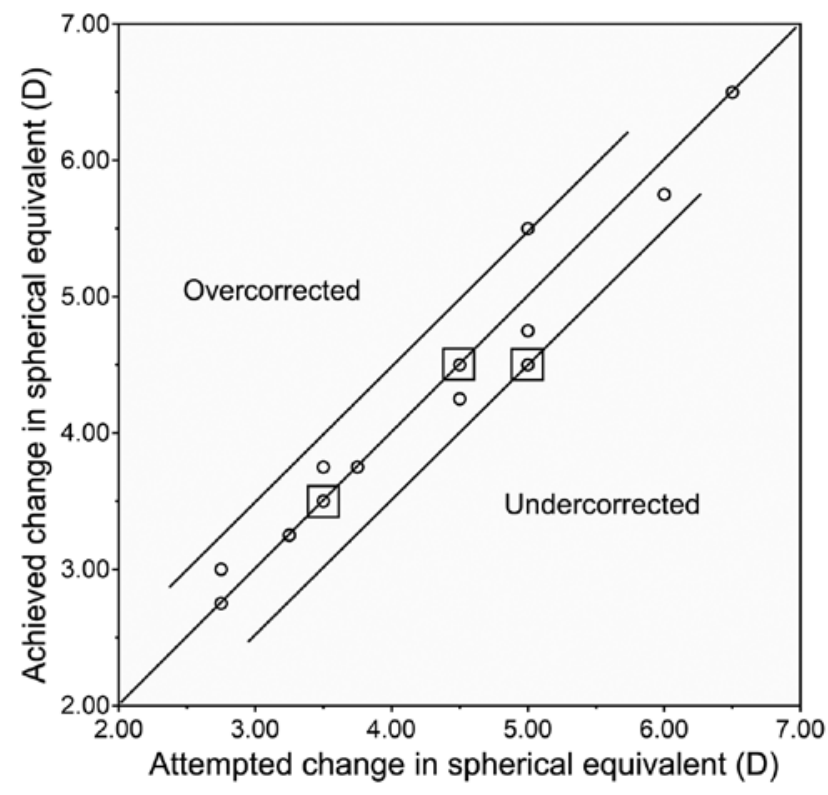

Figure 3. Predictability of outcomes. Scatterplot of the attempted vs. achieved correction 6 months after implantable collamer lens implantation $(\mathrm{R}=0.944$; $\mathrm{y}=0.33+0.91 \mathrm{x})$. The points enclosed by the squares represent the locations of multiple data duplications. D, diopters. implantation is better than corneal refractive surgery for the correction of moderate and high myopia, particularly in terms of the reduced disturbance of night vision (17-19). However, data regarding the consequences of ICLs with a central hole for residual refractive error correction after corneal refractive surgery are scarce. The aim of the present study was to evaluate the efficacy, safety, predictability and quality of vision in patients who underwent posterior chamber ICL V4c implantation for residual refractive error after corneal refractive surgery.

In the present study, an improvement in UCVA was obtained after ICL V4c implantation for residual refractive error correction after corneal refractive surgery, and at 6 months after the second surgery, all eyes gained a UCVA of 20/20 or better. The efficacy index was 1.19 at 6 months post-operatively, which was good, with a BCVA of $-0.02 \pm 0.07$. An effective index greater than or equal to 1 indicates that the surgery was effective. The safety index was 1.26 . In our study, most eyes maintained the BCVA, and some gained more lines of BCVA, which was consistent with findings from previous case reports $(18,19)$. A safety index $\geq 1$ indicates that the operation is safe and acceptable. In the follow-up period, none of the eyes lost $>1$ line of BCVA. The predictability was also high; $100 \%$ of eyes were within $\pm 0.50 \mathrm{D}$ of the predicted refractive 
change. At post-operative 6 months, the MRSE of all patients was near emmetropia $(\mathrm{R}=0.944)$.

In agreement with those of previous studies $(20,21)$, the present study demonstrated good results obtained with ICL for residual refractive error correction after corneal refractive surgery and provided a contribution to accurate pre-operative optometry. In the implantation of posterior-chamber ICLs, the calculation appeared less dependent on corneal refractive power but more on precise subjective refraction. The reliability of autorefractometry after corneal refractive surgery is influenced by the pre-operative amount of myopia and the laser optic ablation zone (22). Since the cornea becomes more flattened after laser surgeries, there are more possibilities of positive spherical aberration, which results from different focusing of the beams on the peripheral and central part of an optical system (23). When increasing the positive spherical aberration after corneal refractive surgery, higher refractive power are encountered in the pupil area by peripheral imaging rays, where the retinal image form appears to be more myopic compared with subjective refraction (22). In consideration of these factors, reasonable adjustments were made during the design of the operation plan according to age and previous surgical history.

Furthermore, the present study indicated that there was no significant difference in the OSI, SR and the OVs among the optical quality parameters at contrasts of $9 \%$, pre-operatively and post-operatively. However, significant changes of the MTF cutoff frequency were obtained in the present study, as well as OVs at contrasts of 100 and $20 \%$ prior to and after surgery. The OVs at contrasts of $100 \%$ were directly related to the MTF cutoff frequency (it is the MTF cutoff frequency divided by 30 cycles/degree), which reflects the patients' vision during the day. In the present study, there were significant differences in the MTF cutoff frequencies and OVs at contrasts of 100 and $20 \%$, which indicated that ICL implantation for correcting residual myopia contributed to good visual quality mainly during the daytime. ICL implantation does not involve surgical tissue abstraction and leaves the central cornea untouched; therefore, the visual quality was essentially improved post-operatively. However, the night vision loss due to corneal refractive surgery cannot be improved. In the present study, all patients had undergone corneal refractive surgeries a few years earlier and had increased cornea spherical aberration, which partly contributed to the changes in contrasts of $9 \%$ OVs after ICL implantation. After the corneal refractive surgeries, the corneal total higher-order aberrations (HOAs) and spherical aberration are usually increased $(24,25)$, which may lead to greater intraocular scattering after the second operation and affect the visual quality at contrasts of $9 \%$. OVs at contrasts of $9 \%$ simulate night vision, which may be disturbed after corneal laser refractive surgery, including PRK or LASIK, which has been reported to be the main factor affecting night vision due to increased spherical aberration (25-27).

Compared with that used in previous studies (28-30), a novel type of ICL with a central hole, without pre-operative peripheral iridotomy, was used in the present study, and good results were also obtained. It was previously reported that the presence of a central hole ICL implantation provided an excellent visual performance almost equivalent to that obtained with conventional ICLs (14). In addition, to evaluate the objective visual quality post-operatively, an OQAS, an advanced tool for quantitatively evaluating optical quality changes after refractive surgeries, was used in the present study. According to the present results, the implantation of ICL with a central hole did not induce a significant additional change in the subjective intraocular forward scattering. These results were in agreement with those of previous studies, which demonstrated that ICL implantation resulted in almost no interference with the visual quality, as there was no statistically significant change in OSI after surgery $(14,18,31)$. Implantation of ICL with central hole significantly improved the optical quality, including the MTF cutoff frequency and OVs at contrasts of 100 and $20 \%$ in a clinical setting. It is therefore indicated that this is a good surgical option for the correction of residual refractive error after corneal refractive surgeries.

There were several limitations to this study. First, the sample size of the present study was relatively small from a statistical viewpoint and the follow-up time was short. If the thickness of the cornea was sufficient, most patients chose laser surgery for re-correction, so the number of patients who used ICL implantation for secondary surgery was relatively small. Another limitation was that the pre-operative and post-operative corneal total HOAs and spherical aberration, which are important factors for evaluating quality of vision, were not assessed. In addition, the subjective quality of vision was not comprehensively evaluated, e.g. by administering a patient questionnaire for night vision.

In conclusion, the present study demonstrated significantly improved optical quality parameters, such as the MTF cutoff frequency, SR, OSI and OVs at contrasts of 100, 20 and 9\%, for patients after corneal refractive surgery undergoing ICL implantation. These results suggested that ICL implantation achieved encouraging results in the correction of residual refractive errors after corneal refractive surgery. Further studies are required to assess patient satisfaction and evaluate subjects for longer post-operative follow-up periods to confirm the safety of the procedure.

\section{Acknowledgements}

Not applicable.

\section{Funding}

This work was partially supported by the Cross-disciplinary Research Fund of Shanghai Ninth People's Hospital, Shanghai Jiao Tong University School of Medicine (grant no. JYJC201907). The funding body had no role in the design or conduct of the study, the collection, management, analysis or interpretation of the data, the preparation, review or approval of the manuscript or in the decision to submit the manuscript for publication.

\section{Availability of data and materials}

The datasets used and/pr analyzed during the current study are available from the corresponding author on reasonable request. 


\section{Authors' contributions}

$\mathrm{XQF}$ analyzed and interpreted the patient data regarding the patients. FLH and YL performed the ocular examination. JZ was a major contributor in writing the manuscript and performed all surgeries. All authors read and approved the final manuscript.

\section{Ethics approval and consent to participate}

The present study was approved by the ethics committee of The Ninth Hospital of Jiao Tong University (Shanghai, China). It followed the tenets of the Declaration of Helsinki. Written informed consent was obtained from all patients after the possible consequences of the study were explained.

\section{Patient consent for publication}

Not applicable.

\section{Competing interests}

The authors declare that they have no competing interests.

\section{References}

1. Jiménez-Alfaro I, Gómez-Tellería G, Bueno JL and Puy P: Contrast sensitivity after posterior chamber phakic intraocular lens implantation for high myopia. J Refract Surg 17: 641-645, 2001.

2. Uusitalo RJ, Aine E, Sen NH and Laatikainen L: Implantable contact lens for high myopia. J Cataract Refract Surg 28: 29-36, 2002.

3. Lackner B, Pieh S, Schmidinger G, Hanselmayer G, Dejaco-Ruhswurm I, Funovics MA and Skorpik C: Outcome after treatment of ametropia with implantable contact lenses. Ophthalmology 110: 2153-2161, 2003

4. Kamiya K, Shimizu K, Igarashi A, Hikita F and Komatsu M Four-year follow-up of posterior chamber phakic intraocular lens implantation for moderate to high myopia. Arch Ophthalmol 127: 845-850, 2009

5. Moshirfar M, Jehangir N, Fenzl CR and McCaughey M: LASIK enhancement: Clinical and surgical management. J Refract Surg 33 116-127, 2017.

6. Schallhorn SC, Venter JA, Hannan SJ, Hettinger KA and Teenan D: Flap lift and photorefractive keratectomy enhancements after primary laser in situ keratomileusis using a wavefront-guided ablation profile: Refractive and visual outcomes. J Cataract Refract Surg 41: 2501-2512, 2015.

7. Siedlecki J, Luft N, Kook D, Wertheimer C, Mayer WJ, Bechmann M, Wiltfang R, Priglinger SG, Sekundo W and Dirisamer M: Enhancement after myopic small incision lenticule extraction (SMILE) using surface ablation. J Refract Surg 33 513-518, 2017.

8. Solaiman KA, Fouda SM, Bor'i A and Al-Nashar HY: Photorefractive keratectomy for residual myopia after myopic laser in situ keratomileusis. J Ophthalmol 2017: 8725172, 2017.

9. Sanders DR, Doney K and Poco M; ICL in Treatment of Myopia Study Group: United States food and drug administration clinical trial of the implantable collamer lens (ICL) for moderate to high myopia: Three-year follow-up. Ophthalmology 111: 1683-1692, 2004.

10. Alfonso JF, Baamonde B, Fernández-Vega L, Fernandes P, González-Méijome JM and Montés-Micó R: Posterior chamber collagen copolymer phakic intraocular lenses to correct myopia: Five-year follow-up. J Cataract Refract Surg 37: 873-880, 2011.

11. Moya T, Javaloy J, Montés-Micó R, Beltrán J, Muñoz G and Montalbán R: Implantable collamer lens for myopia: Assessment 12 years after implantation. J Refract Surg 31: 548-556, 2015.

12. Dougherty PJ and Priver T: Refractive outcomes and safety of the implantable collamer lens in young low-to-moderate myopes. Clin Ophthalmol 11: 273-277, 2017.
13. Kamiya K, Shimizu K, Igarashi A, Kitazawa Y, Kojima T, Nakamura T, Oka Y and Matsumoto R: Posterior chamber phakic intraocular lens implantation: Comparative, multicentre study in 351 eyes with low-to-moderate or high myopia. Br J Ophthalmol 102: $177-181,2018$

14. Kamiya K, Shimizu K, Saito A, Igarashi A and Kobashi H: Comparison of optical quality and intraocular scattering after posterior chamber phakic intraocular lens with and without a central hole (Hole ICL and Conventional ICL) implantation using the double-pass instrument. PLoS One 8: e66846, 2013.

15. Güell JL, Pujol J, Arjona M, Diaz-Douton F and Artal P: Optical quality analysis system; instrument for objective clinical evaluation of ocular optical quality. J Cataract Refract Surg 30: 1598-1599, 2004.

16. Saad A, Saab M and Gatinel D: Repeatability of measurements with a double-pass system. J Cataract Refract Surg 36: 28-33, 2010.

17. Sanders DR: Matched population comparison of the visian implantable collamer lens and standard LASIK for myopia of -3.00 to -7.88 diopters. J Refract Surg 23: 537-552, 2007.

18. Liu HT, Zhou Z, Luo WQ, He WJ, Agbedia O, Wang JX, Huang JZ, Gao X, Kong M, Li M and Li L: Comparison of optical quality after implantable collamer lens implantation and wavefront-guided laser in situ keratomileusis. Int J Ophthalmol 11: 656-661, 2018.

19. Kamiya K, Shimizu K, Igarashi A and Kawamorita T: Effect of myopic defocus on visual acuity after phakic intraocular lens implantation and wavefront-guided laser in situ keratomileusis. Sci Rep 5: 10456, 2015.

20. Kamiya K and Shimizu K: Implantable collamer lens for hyperopia after radial keratotomy. J Cataract Refract Surg 34: 1403-1404, 2008.

21. Srinivasan S, Drake A and Herzig S: Early experience with implantable collamer lens in the management of hyperopia after radial keratotomy. Cornea 27: 302-304, 2008.

22. Mirshahi A, Wesemann W, Bühren J and Kohnen T: Factors influencing the reliability of autorefractometry after LASIK for myopia and myopic astigmatism. Am J Ophthalmol 150: 774-779, 2010.

23. Gatinel D, Adam PA, Chaabouni S, Munck J, Thevenot M, Hoang-Xuan T, Pieger S, Fujieda M and Bains HS: Comparison of corneal and total ocular aberration before and after myopic LASIK. J Refract Surg 26: 333-340, 2010.

24. Sarkar S, Bharadwaj SR, Reddy JC and Vaddavalli PK: Longitudinal changes in optical quality, spatial vision, and depth vision after laser refractive surgery for myopia. Optom Vis Sci 97: 360-369, 2020.

25. Lee K, Ahn JM, Kim EK and Kim TI: Comparison of optical quality parameters and ocular aberrations after wavefront-guided laser in-situ keratomileusis versus wavefront-guided laser epithelial keratomileusis for myopia. Graefes Arch Clin Exp Ophthalmol 251: 2163-2169, 2013.

26. Kung JS and Manche EE: Quality of vision after wavefront-guided or wavefront-optimized LASIK: A prospective randomized contralateral eye study. J Refract Surg 32: 230-236, 2016.

27. Anera RG, Castro JJ, Jiménez JR, Villa C and Alarcón A: Optical quality and visual discrimination capacity after myopic LASIK with a standard and aspheric ablation profile. J Refract Surg 27: 597-601,2011.

28. Chen X, Wang XY, Zhang X, Chen Z and Zhou XT: Implantable collamer lens for residual refractive error after corneal refractive surgery. Int J Ophthalmol 9: 1421-1426, 2016.

29. Lee J, Kim Y, Park S, Bae J, Lee S, Park Y, Lee J and Lee JE: Long-term clinical results of posterior chamber phakic intraocular lens implantation to correct myopia. Clin Exp Ophthalmol 44: 481-487, 2016.

30. Pjano MA, Pidro A, Biscevic A, Grisevic S, Pandzic B and Cerovic V: Refractive outcomes of posterior chamber phakic intraocular lens implantation for correction of myopia and myopic astigmatism. Med Arch 71: 93-96, 2017.

31. Iijima A, Shimizu K, Yamagishi M, Kobashi H, Igarashi A and Kamiya K: Assessment of subjective intraocular forward scattering and quality of vision after posterior chamber phakic intraocular lens with a central hole (Hole ICL) implantation. Acta Ophthalmol 94: e716-e720, 2016.

This work is licensed under a Creative Commons Attribution-NonCommercial-NoDerivatives 4.0 International (CC BY-NC-ND 4.0) License. 